\title{
Projetos de Vida na Adolescência: uma Revisão Sistemática da Literatura
}

\section{Life Purpose in Adolescence: a Systematic Review of Literature}

\author{
Gabriela Pagano Vieira' (orcid.org/0000-0003-0309-6864) \\ Letícia Lovato Dellazzana-Zanon² (orcid.org/ 0000-0003-0649-1675)
}

\begin{abstract}
Resumo
A adolescência é um período do desenvolvimento profícuo para construção de projetos de vida. Sabe-se que adolescentes que têm projetos de vida são mais felizes e são menos propensos a se envolverem em comportamentos de risco. A fim de analisar as pesquisas realizadas com o tema projetos de vida de adolescentes, realizou-se uma revisão sistemática da literatura nacional no período de 2004-2014, a partir das bases de dados vinculadas à BVS-Psi. Identificaram-se 275 artigos, dos quais 12 foram analisados. Observou-se uma prevalência de estudos qualitativos, com uso expressivo de entrevistas semidirigidas e oficinas, e poucos artigos utilizando metodologia quantitativa. Esse resultado está de acordo com a literatura, que indica pouco uso de escalas e questionários para estudar o projeto de vida de adolescentes, apontando para a necessidade de propor instrumentos quantitativos que possam avaliar projetos de vida em grandes populações de adolescentes.
\end{abstract}

Palavras-chave: Revisão Sistemática. Projetos de Vida. Adolescentes.

\begin{abstract}
Adolescence is a period of fruitful development for building life purposes. It is known that adolescents who have life purposes are happier and are less likely to engage in risky behaviors. In order to analyze the research carried out with the theme of adolescents' life purposes, a systematic review of the national literature was carried out in the period 2004-2014, based on the databases linked to the BVS-Psi. A total of 275 articles were identified, of which 12 were analyzed. There was a prevalence of qualitative studies, with expressive use of semi-directed interviews and workshops, and few articles using quantitative methodology. This result is in accordance with the literature, which indicates little use of scales and questionnaires to study the life purposes of adolescents, pointing to the need to propose quantitative instruments that can evaluate life purposes in large populations of adolescents.
\end{abstract}

Keywords: Systematic Review. Life purpose. Adolescents.

\footnotetext{
1 Pontifícia Universidade Católica de Campinas, Campinas, Brasil. E-mail: gabrielapaganov@gmail.com. 2 Pontifícia Universidade Católica de Campinas, Campinas, Brasil. E-mail: leticiadellazzana@gmail.com.
} 


\section{Introdução}

A adolescência tem sido considerada como um período propício do desenvolvimento humano para a construção de projetos de vida desde os estudos de autores clássicos da Psicologia, como Piaget (1964/2007) e Erikson (1968/1976). Apesar dessa constância no que se refere à fase primordial para o desenvolvimento do projeto de vida, o mesmo não ocorre em relação ao que se entende por adolescência. Nesse sentido, sabe-se que a adolescência foi, por um longo período, considerada como uma fase conturbada do desenvolvimento, marcada por crises, desequilíbrios e conflitos naturais (Erikson, 1968/1976; Hall, 1904). Entretanto, autores contemporâneos afirmam que essa visão precisa ser questionada, pois tormentos e perturbações não devem ser características de indivíduos que passam por essa fase de desenvolvimento (Damon, 2004; Lerner \& Benson, 2003; Senna \& Dessen, 2012).

Neste estudo, compreende-se a adolescência como "um período de evolução e maturação, da passagem de habilidades cognitivas e experiências emocionais menos desenvolvidas da infância para, idealmente, habilidades cognitivas totalmente desenvolvidas e experiências da idade adulta emocionalmente mais equilibradas" (Organização Pan-Americana da Saúde - Opas, 2003, p. 212). Nesse sentido, entende-se que as habilidades cognitivas, comportamentais e sociais do adolescente podem contribuir ativamente e, muitas vezes, efetivamente para suas próprias mudanças no desenvolvimento (Lerner, Lerner \& Benson,
2011). Assim, ao contrário de se compreender a adolescência como um período negativo do desenvolvimento humano, entende-se essa fase como um momento de possibilidades e de direcionamento em relação à vida adulta.

Da mesma forma como ocorre com a adolescência, definir projeto de vida também é uma tarefa desafiadora. Isso ocorre em função das diferentes perspectivas teóricas que embasam os estudos realizados sobre projetos de vida (Dellazzana-Zanon \& Freitas, 2015). Uma das definições mais usadas, sobretudo no contexto internacional, é a proposta por Damon, Menon e Bronk (2003): o projeto de vida é uma intenção estável e generalizada de alcançar algo que seja significativo para o indivíduo e que gere um compromisso para o mundo além do eu. No entanto, alguns autores pontuam que, não necessariamente, o projeto de vida precisa ser significativo para a sociedade (Miranda \& Alencar, 2011, 2015).

Quanto à importância do projeto de vida, sabe-se que ter um projeto de vida pode ser visto como um fator de proteção para o adolescente (Bronk, 2014; Damon, 2009; Mariano \& Going, 2011). Da mesma forma, a ausência de um projeto de vida pode estar relacionada a comportamentos de risco (Bronk, 2014; Costa \& Assis, 2006; Damon, 2009). Isso ocorre porque os riscos frequentes à vida dos adolescentes, como uso de drogas e comportamento sexual de risco, estão vinculados a uma perspectiva pessoal de futuro frágil ou inexistente, como se a vida não valesse a pena (Costa \& Assis, 2006). Desse modo, o foco na construção do projeto de vida poderia evitar que 
os adolescentes se envolvessem em comportamentos autodestrutivos.

Além de ser um fator de proteção, pesquisas afirmam que ter um projeto de vida é um fator que contribui para o desenvolvimento juvenil positivo (Mariano \& Going, 2011), abordagem recente que tem se destacado, sobretudo na literatura internacional, por considerar adolescentes e jovens como recursos com potencialidades que podem ser observadas, reconhecidas e aprimoradas (Damon, 2004; Mariano \& Going, 2011). Essa visão é importante, pois: a) rompe de forma explícita com a ideia de que a adolescência é uma fase turbulenta e problemática do desenvolvimento e b) oferece a possibilidade de ver esse período do desenvolvimento de forma mais positiva e saudável (Dellazzana-Zanon, Bachert \& Gobbo, 2018).

A abordagem do desenvolvimento juvenil positivo parte do princípio de que o processo básico de desenvolvimento humano envolve influências mútuas e relacionadas entre o desenvolvimento individual do sujeito e seu contexto (Lerner, Lerner \& Benson, 2011). Uma das premissas dessa abordagem é que existe uma influência mutua entre o ambiente e a pessoa, e quando essa relação é benéfica para ambas as partes, pode-se considerar que ocorre uma regulação adaptativa do desenvolvimento . Não por acaso, o projeto de vida é um dos aspectos considerados nos estudos sobre desenvolvimento juvenil positivo.

Mariano e Going (2011) desenvolveram um estudo no qual relacionaram diversos indicadores de desenvolvimento juvenil positivo com projetos de vida bem estruturados. Os resultados desse estudo mostraram que o projeto de vida é um dos fatores que promove o desenvolvimento juvenil positivo. Portanto, existem evidências de que ter um projeto de vida fornece suporte para enfrentar situações adversas que os indivíduos costumam vivenciar na adolescência (Mariano \& Going, 2011). Sabese ainda que, para a maioria dos adolescentes, ter um projeto de vida faz com que eles se sintam bem, pois essa perspectiva de finalidade traz para o jovem uma visão mais clara e conciliatória entre presente e futuro (Mariano \& Going, 2011).

Considerando-se que: a) o projeto de vida assume grande importância durante a adolescência, b) que o projeto de vida é um dos fatores que promove o desenvolvimento juvenil positivo, e c) que é necessário para futuras pesquisas saber se e de que forma os pesquisadores nacionais estão estudando projetos de vida, este estudo tem como objetivo identificar e analisar a) os objetivos dos estudos empíricos nacionais sobre projetos de vida de adolescentes, b) os delineamentos de pesquisa usados nos estudos nacionais sobre o tema, c) descrever os instrumentos utilizados para avaliar projetos de vida de adolescentes e d) se existem e quais são os instrumentos quantitativos usados para acessar projetos de vida de adolescentes. Assim, seguindo a orientação de Sampaio e Mancini (2007) para a realização de revisões sistemáticas de literatura, a pergunta de pesquisa deste estudo é: quais são os objetivos, os delineamentos e os instrumentos dos estudos nacionais produzidos entre 2004 e 2014 sobre projetos de vida de adolescentes?

\section{Método}


O estudo foi desenvolvido por meio de uma revisão sistemática de literatura. Para fins da realização deste trabalho, duas pesquisadoras avaliaram, de forma independente, cada um dos artigos selecionados. A busca dos artigos foi feita na Biblioteca Virtual em Saúde Psicologia (BVSPsi), utilizando as bases de dados: a) Index Psi Periódicos Técnico-Científicos, b) Periódicos Eletrônicos em Psicologia (PePSIC), e c) Scientific Eletronic Library Online (SciELO). Utilizaram-se os descritores: a) "projeto de vida" ou "projetos de vida" ou "projeto vital" ou "projetos vitais" e b) "adolescência" ou "adolescente" ou "adolescentes".

Os descritores poderiam estar no título, nas palavras-chaves, no resumo ou no texto em si. Incluíram-se apenas os artigos no qual o estudo de projeto de vida fosse o tema principal, não somente um resultado do estudo. Assim, os critérios de inclusão para o presente artigo foram: a) estudos empíricos nacionais, b) disponíveis online na íntegra, c) com participantes adolescentes, d) cujo projeto de vida fosse o tema central do estudo e e) que fossem publicados entre os anos 2004 a 2014.

\section{Resultados e Discussão}

Inicialmente, foram encontrados 275 artigos. Descartando-se os repetidos (183), restaram 92 artigos. Eliminaram-se 16 artigos por serem sobre gravidez na adolescência e seis por terem participantes de outras fases do ciclo vital. Como este estudo tem como objetivo analisar apenas estudos brasileiros, eliminou-se um artigo em espanhol. Desse modo, restaram 69 artigos, dos quais foram lidos os resumos para verificar se projeto de vida era o tema central do estudo. Eliminaram-se 56 artigos que não tinham projeto de vida como tema central, ou seja, artigos que apenas citavam projeto de vida ou que não buscavam estuda-lo. Além disso, também foi descartado um artigo de caráter documental, uma vez que a presente pesquisa tinha como objetivo estudar os instrumentos utilizados para conhecer o projeto de vida de adolescentes. Assim, foram analisados 12 artigos. A Figura 1 ilustra o processo de definição dos artigos a serem analisados neste estudo.

Figura 1. Percurso metodológico do levantamento bibliográfico

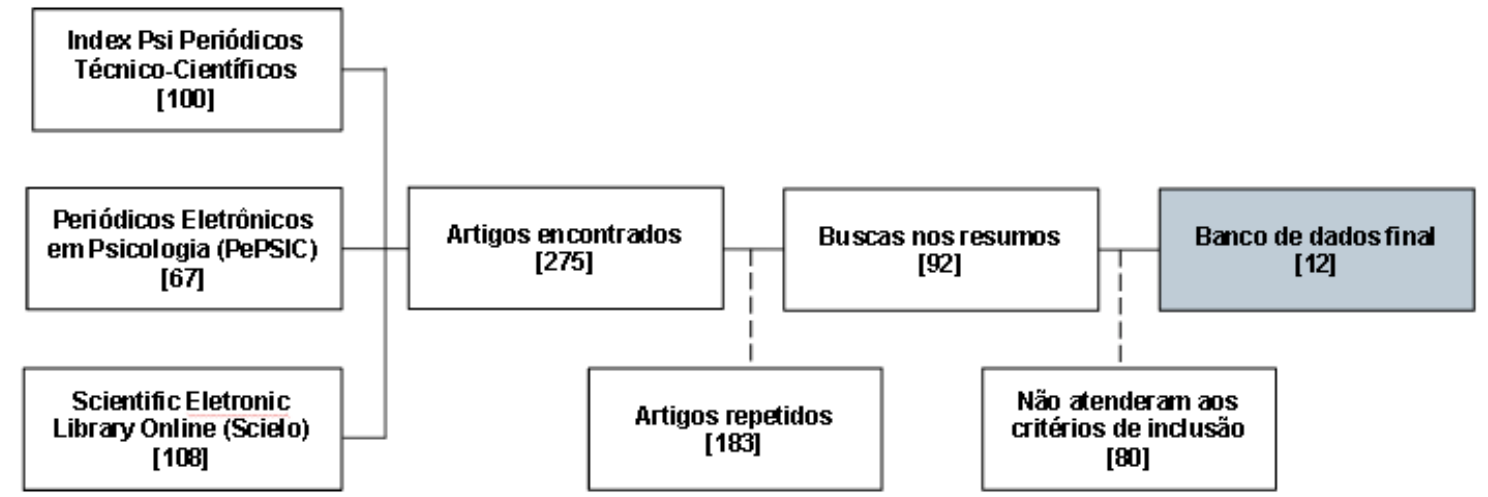

Fonte: Elaborada pelos autores. 
Os 12 estudos analisados foram publicados entre os anos 2006 a 2014. Embora os artigos tivessem objetivos distintos, observouse que os pesquisadores estavam interessados em investigar: a) como o adolescente compreende projeto de vida, b) o que ele pensa sobre esse construto e c) qual a influência da escola ou do trabalho no projeto de vida. Alguns estudos também abordaram a questão da orientação profissional e sua ligação com o projeto de vida dos adolescentes. Assim, no que se refere aos objetivos dos estudos encontrados, foi possível agrupá-los em: a) significados atribuídos ao projeto de vida pelos adolescentes, b) projeto de vida como parte da orientação profissional e c) influência de medidas socioeducativas no projeto de vida dos jovens. A seguir são apresentados os resultados específicos de cada conjunto de objetivos.

Significados Atribuídos ao Projeto de Vida pelos Adolescentes

Observou-se que, dos 12 artigos selecionados, seis tinham como objetivo conhecer a opinião de adolescentes sobre seus projetos de vida e os significados atribuídos a estes (Borges \& Coutinho, 2010; D’AureaTardeli, 2008; Gonçalves et al., 2008; Locatelli, Bzuneck \& Guimarães, 2007; Marcelino, Catão \& Lima, 2009; Nascimento, 2006). Nascimento (2006) investigou os principais significados que adolescentes com idades entre 15 e 24 anos, de escolas públicas de Belém/PA, colocam em seus projetos de vida e a importância que eles atribuem à escola para a realização desses projetos.
A pesquisa de Locatelli, Bzuneck e Guimarães (2007) procurou compreender se adolescentes de 15 a 19 anos, de escolas públicas e particulares de Londrina/PR, percebem os estudos como um meio de alcançarem suas metas profissionais e quais são suas motivações e perspectivas de tempo futuro. $\mathrm{O}$ estudo de Gonçalves et al. (2008) buscou conhecer representações sociais de jovens de 13 a 22 anos, de Duque de Caxias/RJ, acerca dos problemas, suas formas de enfrentamento e como essas representações organizam seu projeto de vida, ou seja, quais seus principais obstáculos na concretização de seus projetos.

D'Aurea-Tardeli (2008) investigou o perfil de jovens de 16 a 18 anos, de escolas particulares do estado de São Paulo/SP, analisando como as variáveis pessoais incidem na consciência do que eles pensam que deve ter feito e nos seus projetos de vida. O estudo de Marcelino, Catão e Lima (2009) comparou as representações sociais de adolescentes com idades entre 16 e 19 anos provenientes de escolas públicas e particulares de João Pessoa/PB, a respeito de seus projetos de vida e de sua construção. Por fim, Borges e Coutinho (2010) pesquisaram a experiência profissional de jovens e buscaram compreender o sentindo que jovens com idades entre 15 e 17 anos, participantes de uma ONG em Florianópolis/SC, dão para o trabalho e como projetam seus caminhos futuros.

\section{Projeto de Vida como Parte da Orientação Profissional}

Dos 12 artigos selecionados, quatro tinham como objetivo investigar o projeto de 
vida como parte da orientação profissional (Becker, Bobato \& Schulz, 2012; Costa, 2007; Titon, Urnau \& Zanella, 2006; Valore \& Viaro, 2007). Valore e Viaro (2007) investigaram as expectativas em relação ao projeto de vida de adolescentes com idades entre 15 e 18 anos, participantes de um projeto de orientação profissional em Curitiba/PR. O estudo de Titon, Urnau e Zanella (2006) buscou criar um espaço de reflexão e de rupturas com as marcas e vícios impressos pela cotidianidade, por meio de uma oficina feita com adolescentes de 14 a 16 anos, em uma escola pública em Florianópolis/SC, na qual o foco foi orientação profissional e projeto de vida.

Costa (2007) realizou um projeto de orientação profissional para jovens com idades entre 13 e 18 anos, de uma comunidade de Belo Horizonte/MG, e teve como objetivo fornecer um espaço de reflexão sobre as possibilidades de estudo e de futuras profissões. Por fim, a pesquisa de Becker, Bobato e Schulz (2012) teve como objetivo promover a reflexão de adolescentes com idades entre 14 e 17 anos, de escolas públicas de Itajaí/SC, sobre projeto de vida e iniciação profissional, por meio de oficinas semanais analisadas pela abordagem sócio-histórica. Deve-se destacar que um objetivo comum observado nesses quatro estudos foi a análise de oficinas de orientação profissional, nas quais o projeto de vida era um tema abordado e, futuramente, estudado pelos pesquisadores.

Influência de Medidas Socioeducativas no Projeto de Vida dos Jovens
O terceiro grupo com temática semelhante encontrado na busca (dois artigos) teve como objetivo estudar como a medida socioeducativa interfere no projeto de vida do jovem infrator (Gomes \& Conceição, 2014; Souza Neto \& Centolanza, 2010). A pesquisa de Souza Neto e Centolanza (2010) foi sobre um programa de medidas socioeducativas que atende jovens nessa situação em municípios da grande São Paulo/SP e abordou, de forma específica, o caso de dois adolescentes, buscando compreender suas histórias, seus delitos e também a ressignificação de seus projetos de vida. Já o estudo de Gomes e Conceição (2014) buscou compreender o significado que adolescentes de 15 a 20 anos, de Brasília/DF, dão para a medida de liberdade assistida, utilizando como base a teoria socioeconômica. Observou-se que ambas as pesquisas (Gomes \& Conceição, 2014; Souza Neto \& Centolanza, 2010) tiveram em comum o interesse em investigar a eficiência pedagógica das medidas socioeducativas, analisando se ela cumpre com sua função de socializar o jovem, trazendo-o de volta à comunidade e possibilitando a reconstrução de seu projeto de vida.

No que se refere ao segundo objetivo deste estudo, investigar os delineamentos de pesquisa usados nos estudos nacionais sobre projetos de vida, observou-se que, dos 12 artigos selecionados, nove foram qualitativos, dois foram quantitativos e um foi misto. Deve-se ressaltar que o objetivo deste estudo foi investigar os instrumentos que avaliavam de forma específica o projeto de vida de adolescentes. Portanto, os instrumentos utilizados nas pesquisas para acessar outras variáveis não foram analisados. O método e os 
instrumentos utilizados para avaliar o projeto de

vida

estão

expostos

no

Quadro

1.

Quadro 1. Artigos encontrados, seus métodos e seus instrumentos

\begin{tabular}{|c|c|c|}
\hline Artigo & Método & Instrumento para acessar projeto de vida \\
\hline Nascimento (2006) & Qualitativo & Questionário com três perguntas abertas, elaborado pela autora. \\
\hline $\begin{array}{l}\text { Titon, Urnau \& Zanella } \\
\text { (2006) }\end{array}$ & Qualitativo & $\begin{array}{l}\text { Oficinas. Foi trabalhado projeto de vida em um encontro, por meio } \\
\text { de um texto disparador e uma pergunta feita pelas autoras. }\end{array}$ \\
\hline Costa (2007) & Qualitativo & $\begin{array}{l}\text { Oficina de orientação profissional que abordou temáticas como } \\
\text { escola, trabalho e o futuro de adolescentes, sem detalhamento de } \\
\text { instrumentos para acessar o projeto de vida. }\end{array}$ \\
\hline $\begin{array}{l}\text { Locatelli, Bzuneck \& } \\
\text { Guimarães (2007) }\end{array}$ & Quantitativo & $\begin{array}{l}\text { Questionário de autorrelato com escala tipo Likert, elaborado pelos } \\
\text { autores, com quatro questões fechadas abordando o projeto de vida. }\end{array}$ \\
\hline Valore \& Viaro (2007) & Quantitativo & $\begin{array}{l}\text { Questionário com quatro perguntas abertas e uma fechada, } \\
\text { elaborado pelas autoras, sobre os projetos de vida dos adolescentes. }\end{array}$ \\
\hline D'Aurea-Tardeli (2008) & Misto & $\begin{array}{l}\text { O projeto de vida foi avaliado por } \\
\text { contendo questões elaboradas pela }\end{array}$ \\
\hline Gonçalves et al. (2008) & Qualitativo & $\begin{array}{l}\text { Entrevistas semiestruturadas elaboradas pelas autoras. Algumas } \\
\text { questões abordam o futuro ou o projeto de vida dos adolescentes. }\end{array}$ \\
\hline $\begin{array}{l}\text { Marcelino, Catão \& } \\
\text { Lima (2009) }\end{array}$ & Qualitativo & $\begin{array}{l}\text { Entrevista semiestruturada elaborada pelas autoras, com perguntas } \\
\text { acerca das concepções de projeto de vida dos adolescentes. }\end{array}$ \\
\hline $\begin{array}{l}\text { Borges \& Coutinho } \\
(2010)\end{array}$ & Qualitativo & $\begin{array}{l}\text { Entrevista semiestruturada, elaborada pelas autoras, que buscava } \\
\text { conhecer o adolescente, seu trabalho e seus projetos futuros. }\end{array}$ \\
\hline $\begin{array}{ll}\text { Souza } \quad \text { Neto } \\
\text { Centolanza }(2010)\end{array}$ & Qualitativo & $\begin{array}{l}\text { Entrevistas diretivas e não diretivas, elaboradas pelos autores. Não } \\
\text { foi exposto de maneira detalhada em qual entrevista e como o } \\
\text { projeto de vida foi questionado. }\end{array}$ \\
\hline $\begin{array}{l}\text { Becker, Bobato } \\
\text { Schulz (2012) }\end{array}$ & Qualitativo & $\begin{array}{l}\text { O projeto de vida foi abordado por meio de relato escrito a partir de } \\
\text { questões problematizadoras e por relato oral. }\end{array}$ \\
\hline $\begin{array}{l}\text { Gomes \& Conceição } \\
\text { (2014) }\end{array}$ & Qualitativo & $\begin{array}{l}\text { Oficinas de dramatizações, elaborada pelas autoras. A última oficina } \\
\text { abordou projetos de vida e o futuro dos participantes. }\end{array}$ \\
\hline
\end{tabular}

Fonte: Elaborado pelas autoras. 
Dos nove artigos que empregaram metodologia qualitativa, quatro artigos utilizaram entrevistas semiestruturadas (Borges \& Coutinho, 2010; Gonçalves et al., 2008; Marcelino, Catão \& Lima, 2009; Souza Neto \& Centolanza, 2010), quatro artigos utilizaram oficinas (Becker, Bobato \& Schulz, 2012; Costa, 2007; Gomes \& Conceição, 2014; Titon, Urnau \& Zanella, 2006) e um estudo utilizou questionário (Nascimento, 2006). Dos artigos que utilizaram entrevistas semiestruturadas, dois apresentavam um roteiro condutor para desenvolver a entrevista (Borges \& Coutinho, 2010; Gonçalves et al., 2008;). Outro estudo apresentou uma entrevista diretiva e não diretiva (Souza Neto \& Centolanza, 2010).

O estudo de Marcelino, Catão e Lima (2009) utilizou um roteiro semiestruturado, com questões sobre dados pessoais, histórico profissional e projetos de vida dos jovens. Em um segundo momento, foi realizada uma entrevista na qual foram analisas as fotos do cotidiano do trabalho produzidas pelos adolescentes ao longo do processo da pesquisa.

Entre os artigos de caráter qualitativo, quatro foram sobre oficinas feitas com adolescentes (Becker, Bobato \& Schulz, 2012; Costa, 2007; Gomes \& Conceição, 2014; Titon, Urnau \& Zanella, 2006). Três delas analisaram as atividades realizadas com os jovens sobre orientação profissional, como falas produzidas pelos adolescentes durante as dinâmicas e atividades realizadas, o material desenvolvido pelos adolescentes nessas oficinas e demais aspectos qualitativos (Becker, Bobato \& Schulz, 2012; Costa, 2007; Titon, Urnau \& Zanella, 2006). No estudo de Gomes e Conceição (2014), os adolescentes que estavam cumprindo medidas socioeducativas foram convidados a fazer uma oficina de dramatizações. Nessa oficina, eles tinham como tarefa criar um personagem em conjunto com os demais e, ao final, fazer uma dramatização do futuro da própria vida e de seus projetos de vida.

Por fim, somente um estudo qualitativo utilizou questionário para conhecer o projeto de vida de adolescentes (Nascimento, 2006). O estudo de Nascimento (2006) apresentou três perguntas abertas, adaptadas da tese da mesma autora, sobre o que os adolescentes pensam a respeito de seu futuro, como se imaginam daqui a 10 anos e qual a contribuição que eles atribuem à escola para a construção de seus projetos de vida. Com os dados obtidos, foi feita uma análise interpretativa do conteúdo, por meio de técnicas de representações sociais.

Das estratégias quantitativas utilizadas pelos estudos brasileiros, foram utilizados questionários para acessar os projetos de vida de adolescentes (Locatelli, Bzuneck \& Guimarães, 2007; Valore \& Viaro, 2007). O estudo de Locatelli, Bzuneck e Guimarães (2007) utilizou um questionário de autorrelato com escala tipo Likert, elaborado pelos autores, com quatro questões perguntando para adolescentes se eles já sabem o que querem fazer profissionalmente no futuro, se estão seguros da decisão e qual importância pessoal de tal carreira. Além disso, esse instrumento foi composto por mais 34 itens criados ou adaptados de instrumentos já existentes, que buscavam avaliar a motivação para estudar, a valorização dos estudos no presente e a importância do estudo para a concretização de metas de vida. É importante destacar que, embora tenha sido usado um instrumento quantitativo, somente quatro 
questões abordavam o projeto de vida; de forma mais específica, o projeto de vida profissional.

$$
\text { O artigo de Valore e Viaro (2007) }
$$

utilizou um questionário com quatro perguntas abertas e uma fechada. No entanto, para a elaboração do artigo, só foram utilizados os enunciados que questionavam a respeito do projeto de vida do adolescente e as contribuições que a profissão que ele pretendia escolher traz para a sociedade. Assim, esse estudo utilizou técnicas quantitativas para analisar e mensurar os dados obtidos com as perguntas que os adolescentes responderam (Valore \& Viaro, 2007). Destaca-se que o questionário usado por Valore e Viaro (2007) não continha medidas quantitativas, tratando-se de perguntas abertas sobre projeto de vida. Cabe também ressaltar que os questionários utilizados nesse estudo eram bastante breves, apresentando apenas quatro questões sobre projeto de vida.

Foi encontrado apenas um estudo de caráter qualitativo e quantitativo (D'AureaTardeli, 2008). Ele utilizou o Prosocial Reasoning Objective Measure (PROM), o qual avalia objetivamente o nível de raciocínio moral pró-social dos adolescentes. Nesse estudo, foram utilizadas três histórias com dilemas de ordem moral que o participante deveria resolver e, de acordo com a resposta, era classificado em categorias de pró-socialidade. Em seguida, o adolescente respondia a uma questão aberta de autorrelato a respeito de como ele se via dali a 10 anos. Essa segunda fase da pesquisa visava relacionar adolescentes com categorias hedonistas no PROM com projetos de vida pouco solidários ou bastante solidários, no caso de adolescentes com valores internalizados. Destaca-se que o material utilizado para obter dados acerca do projeto de vida dos adolescentes é qualitativo, tratando-se de uma pergunta aberta, sendo que o instrumento quantitativo do estudo foi utilizado para mesurar a prósocialidade do jovem. Assim, embora seja um artigo misto, o projeto de vida foi estudado apenas de maneira qualitativa.

Observando o material encontrado na busca, foi possível observar que somente três artigos foram quantitativos (Locatelli, Bzuneck \& Guimarães, 2007; Valore \& Viaro, 2007), sendo que um deles apresentou também instrumento qualitativo (D'Aurea-Tardeli, 2008). Contudo, embora fossem estudos quantitativos, apenas um deles utilizou um instrumento quantitativo para acessar projetos de vida (Locatelli, Bzuneck \& Guimarães, 2007). Sendo assim, o questionário de autorrelato com escala tipo Likert, de Locatelli, Bzuneck \& Guimarães (2007), contendo quatro questões, foi o único instrumento quantitativo utilizado para acessar projetos de vida de adolescentes encontrado na busca de estudos brasileiros. Deve-se ressaltar que o instrumento encontrado apresentou poucas perguntas sobre o projeto de vida, além de enfocar somente o projeto de vida profissional.

Desse modo, pode-se concluir que a maioria dos estudos sobre projeto de vida na adolescência utilizou instrumentos qualitativos, como oficinas e entrevistas semiestruturadas. Esse resultado está em consonância com o estudo de Dellazzana-Zanon \& Freitas (2015), segundo o qual há uma predominância do uso de entrevistas, de questões de autorrelato e de grupos de discussão nas pesquisas brasileiras sobre projetos de vida de adolescentes. Nesse mesmo sentido, Damon (2009) destaca que as 
abordagens metodológicas utilizadas para acessar os projetos de vida dos adolescentes variam bastante, desde explorações qualitativas de diários de adolescentes até respostas mais estruturadas para questões específicas e entrevistas. Assim, este estudo de revisão de literatura indica que, no Brasil, predominam instrumentos de caráter qualitativo para a investigação de projetos de vida de adolescentes.

\section{Considerações Finais}

Este estudo de revisão sistemática de literatura buscou responder à seguinte pergunta: quais são os objetivos, os delineamentos e os instrumentos dos estudos nacionais produzidos entre 2004 e 2014 sobre projetos de vida de adolescentes? Os resultados mostraram que os objetivos dos estudos encontrados podem ser agrupados da seguinte forma: a) significados atribuídos ao projeto de vida pelos adolescentes, b) projeto de vida como parte da orientação profissional e c) influência de medidas socioeducativas no projeto de vida dos jovens.

Quanto aos delineamentos de pesquisa usados nos estudos nacionais sobre projetos de vida de adolescente no período de estudo, os resultados mostraram que existem diferentes maneiras de se estudar projeto de vida em adolescentes brasileiros. Observou-se, no entanto, uma predominância de estudos de caráter qualitativo, sendo os instrumentos, em sua maioria, entrevistas semiestruturadas e oficinas.

Esse resultado chama atenção pela predominância de estudos qualitativos para acessar projetos de vida de adolescentes no contexto brasileiro, que embora sejam importantes para entender como essa população constrói seu projeto de vida, não podem ser aplicados em larga escala, como fazem os instrumentos quantitativos. Sem instrumentos dessa natureza, não é possível estudar grandes populações de adolescentes nem realizar estudos que associem projeto de vida com outras variáveis. Além disso, destaca-se a importância de utilizar instrumentos quantitativos para avaliar intervenções, razão a mais para se pensar na construção de um instrumento que avalie projetos de vida de forma quantitativa.

Deve-se destacar que, por se tratar de um estudo de revisão de literatura, foi necessário definir descritores para a busca nas bases de dados. Assim, é possível que não tenham sido encontrados artigos sobre a temática em questão que não tenham utilizado os descritores escolhidos. Além da limitação originada pelos descritores utilizados, a presente pesquisa buscou investigar apenas estudos nacionais. Desse modo, muitos estudos relevantes para a temática projetos de vida não foram analisados por se tratarem de artigos de outros países.

Considerando-se a importância do estudo dos projetos de vida de adolescentes e a necessidade de se planejar intervenções que promovam projetos de vida nessa faixa etária, sugere-se que estudos futuros invistam na construção de instrumentos quantitativos cujo foco seja acessar projetos de vida de adolescentes brasileiros. Espera-se que esses futuros instrumentos possam ser úteis para avaliar intervenções com o escopo de promover projetos de vida em adolescentes e desenvolvimento juvenil positivo. 


\section{Referências}

Becker, A. P. S., Bobato, S. T., \& Schulz, M. L. C. (2012). Meu lugar no mundo: relato de experiência com jovens em orientação profissional. Revista Brasileira de Orientação Profissional, 13(2), 253-264.

Borges, R. C. P., \& Coutinho, M. C. (2010). Trajetórias juvenis: significando projetos de vida a partir do primeiro emprego. Revista Brasileira de Orientação Profissional, 11(2), 189-200.

Bronk, K. C. (2014). Purpose in Life: a Critical Component of Optimal Youth Development. New York: Springer.

Costa, C. R. B. S. F. da, \& Assis, S. G. de. (2006). Fatores protetivos a adolescentes em conflito com a lei no contexto socioeducativo. Psicologia \& Sociedade, 18(3), 74-81.

Costa, J. M. C. (2007). Orientação profissional: um outro olhar. Psicologia USP, 18(4), 7987.

Damon, W. (2004). What is Positive Youth Development?. Annals of the American Academy of Political and Social Science. Special Issue: Positive Development: Realizing the Potential of Youth, 591, 13-24.

Damon, W. (2009). O que o jovem quer da vida? Como pais e professores podem orientar e motivar os adolescentes (J. Valpassos, Trad.). São Paulo: Summus.

Damon, W., Menon, J., \& Bronk, K. C. (2003). The Development of Purpose during Adolescence. Applied Developmental Science, 7(3), 119-128.

D’Aurea-Tardeli, D. (2008). A manifestação da solidariedade em adolescentes: um estudo sobre a personalidade moral. Psicologia: Ciência e Profissão, 28(2), 288-303.

Dellazzana-Zanon, L. L., Bachert, C. M. D., \& Gobbo, J. P. (2018). Projetos de vida do adolescente: implicações para a escolarização positiva. In T. C Nakano. (Org.). Psicologia positiva aplicada à
Educação (pp. 41-62). São Paulo: Vetor Editora.

Dellazzana-Zanon, L. L., \& Freitas, L. B. L. (2015). Uma revisão de literatura sobre a definição de projeto de vida na adolescência. Interação em Psicologia (Online), 19(2), 281-292.

Erikson, E. H. (1976). Identidade, juventude e crise (A. Cabral, Trad.). Rio de Janeiro: Zahar Editores. (Obra original publicada em 1968).

Gomes, C. C., \& Conceição, M. I. G. (2014). Sentidos da trajetória de vida para adolescentes em medida de liberdade assistida. Psicologia em Estudo, 19(1), 47-58.

Gonçalves, H. S., Borsoi, T. S., Santiago, M. A., Lino, M. V., Lima, I. N., \& Federico, R. G. (2008). Problemas da juventude e seus enfrentamentos: um estudo de representações sociais. Psicologia e Sociedade, 20(2), 217-225.

Hall, G. S. (1904). Adolescence, its Psychology and its Relations to Physiology, Anthropology, Sociology, Sex, Crime, Religion and Education. New York: Appleton and Company.

Lerner, R. M., \& Benson, P. L. (Eds.). (2003). Assets and Asset-Building Communities: Implications for Research, Policy and Practice. New York: Kluwer Academic.

Lerner, R. M., Lerner, J. V., \& Benson, J. B. (2011). Positive Youth Development: Research and Application for Promoting Thriving in Adolescences. In R. M. Lerner, J. V. Lerner \& J. B. Benson (Eds.). Advances in Child Development and Behavior - Positive Youth Development, 41, 117.

Locatelli, A. C. D., Bzuneck, J. A., \& Guimarães, S. E. R. (2007). A motivação de adolescentes em relação com a perspectiva de tempo futuro. Psicologia: Reflexão e Crítica, 20(2), 268-276.

Marcelino, M. Q. S., Catão, M. F. F. M., \& Lima, C. M. P, (2009). Representações sociais do projeto de vida entre adolescentes no ensino médio. Psicologia: Ciência e Profissão, 29(3), 544-557. 
Mariano, J. M., \& Going, J. (2011). Youth Purpose and Positive Youth Development. In R. M. Lerner, J. V. Lerner \& J. B. Benson (Eds.). Advances in Child Development and Behavior - Positive Youth Development, 41, 39-68.

Miranda, F. H. F., \& Alencar, H. M. (2011). Moral e ética: a importância dos projetos de vida. II Congresso de Pesquisas em Psicologia e Educação Moral - Conflito nas instituições educativas: Perigo ou oportunidade?, 2011, Campinas.

Miranda, F. H. F., \& Alencar, H. M. de. (2015). Projetos de vida na adolescência: um estudo na área da ética e da moralidade. Diaphora, 15(2), 27-33.

Nascimento, I. P. (2006). Projeto de vida de adolescentes do ensino médio: um estudo psicossocial sobre suas representações. Imaginário, 12(12), 55-80.

Organización Panamericana de la Salud - OPAS. (2003). Medios y salud: la voz de los adolescentes. Informe Regional (OPS/FCH/CA No.1). Washington, DC: OPS.

Piaget, J. (2007). Seis estudos de Psicologia. (24th ed., M. A. M. D’Amorim \& P. S. L Silva, Trads.). Rio de Janeiro: Forense Universitária. (Obra original publicada em 1964).

Sampaio, R. F., \& Mancini, M. C. (2007). Estudos de revisão sistemática: um guia para síntese criteriosa da evidência científica. Revista Brasileira de Fisioterapia, 11(1), 83-89.

Senna, S. R. C. M., \& Dessen, M. A. (2012). Contribuições das teorias do desenvolvimento humano para a concepção contemporânea da adolescência. Psicologia: Teoria e Pesquisa, 8(1), 101-108.

Souza Neto, J. C., \& Centolanza, C. A. (2010). Da prática do desvio ao protagonismo. Psico, 41(1), 128-136.

Titon, A. P., Urnau, L. C. \& Zanella, A. V. (2006). Jovem, escola e práticas psi: uma intervenção e algumas de suas ressonâncias. Pesquisas e Práticas

Psicossociais, 2(1), 1-14.

Valore, L. A., \& Viaro, R. V. (2007). Profissão e sociedade no projeto de vida de adolescentes em orientação profissional. Revista Brasileira de Orientação Profissional, 8(2), 57-70.

Recebido em: 29/3/2018

Aprovado em: 5/6/2019 